\title{
Pictorial Review of Benign Anal and Perianal Disease
}

D O'Neill, E Carmody, M Lee, E Thornton, M Morrin, Beaumont Hospital, Dublin, Ireland

\section{Benign}

Lipoma Benign tumour consisting of mature white adipocytes. Lipomas of the large intestine are relatively uncommon and typically affect adults in their $5^{\text {th }}$ to $7^{\text {th }}$ decades. [I]
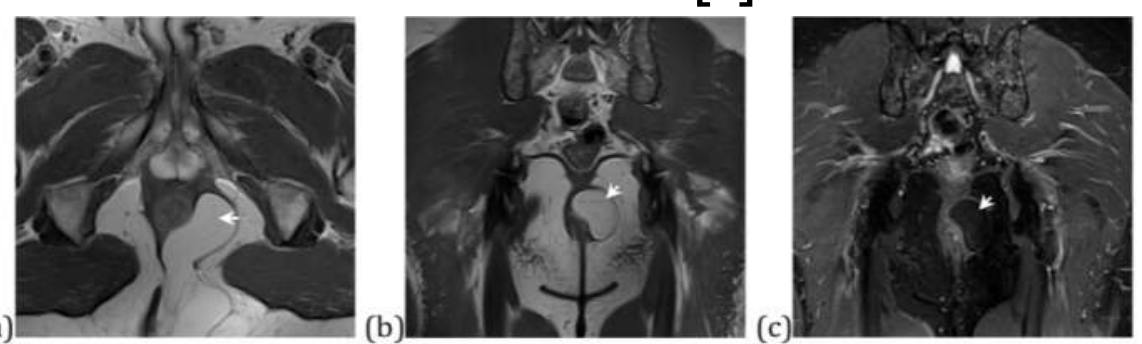

Developmental cysts are lined with epithelium and include epidermoid cysts, dermoid cysts and enteric cysts. Epidermoid cysts are of ectodermal origin arising and are lined with stratified squamous epithelium. [2] Dermoid cysts also arise from the ectoderm. Enteric cysts of endodermal origin.
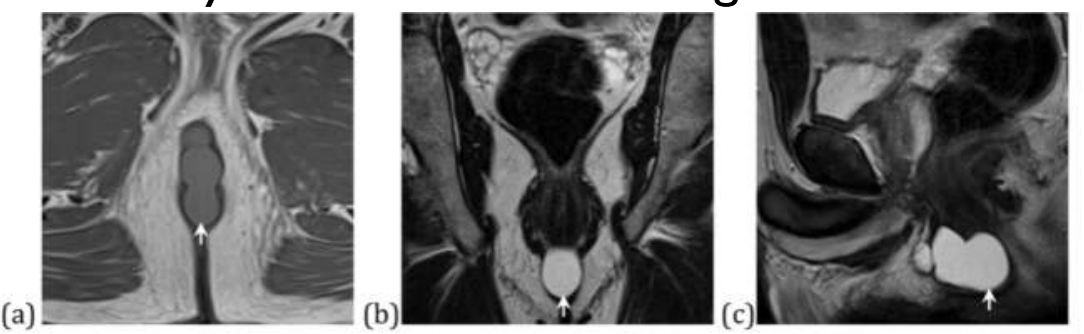

Mucosal prolapse and solitary rectal ulcers syndrome Histological analysis shows replacement of the lamina propria with smooth muscle fibres extending superficially from the muscularis mucosae. [3] It has been suggested that as a result of this dysfunction there is trauma and compression of the anterior rectal wall and upper anal canal with resultant mucosal prolapse [4].
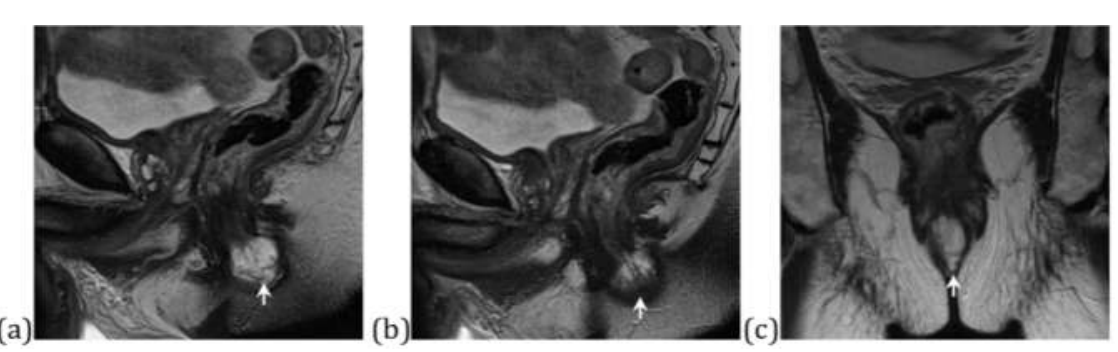

\section{Haemorrhoids}

represent

engorgement of the normal fibrovascular cushions lining the anal canal.

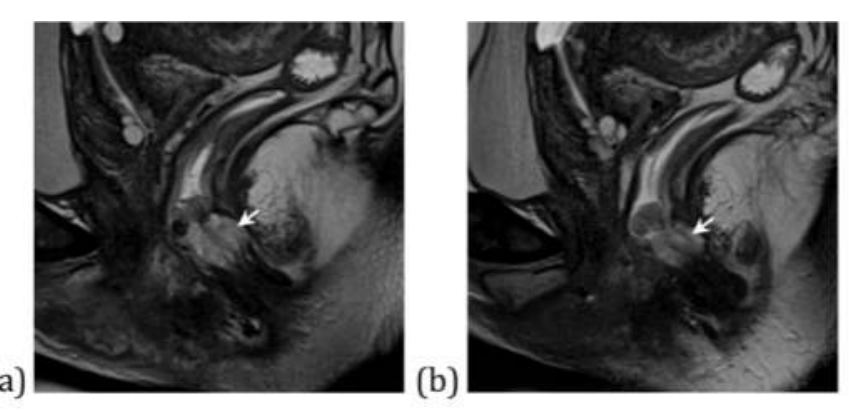

Tarlov Cyst Tarlov cysts are extradural perineural cysts.
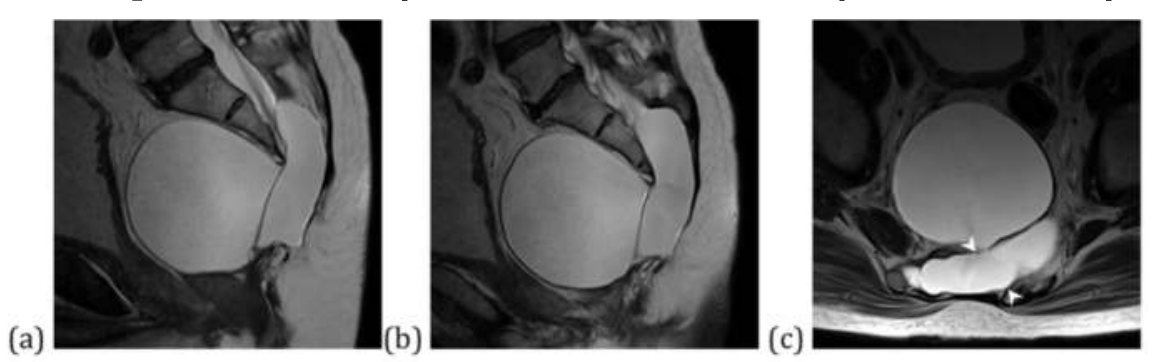

Lesions associated with infection and inflammation: Anorectal Abscess can form in any of the spaces demonstrated in (a), with perianal (b \& c) and ischioanal being most common.
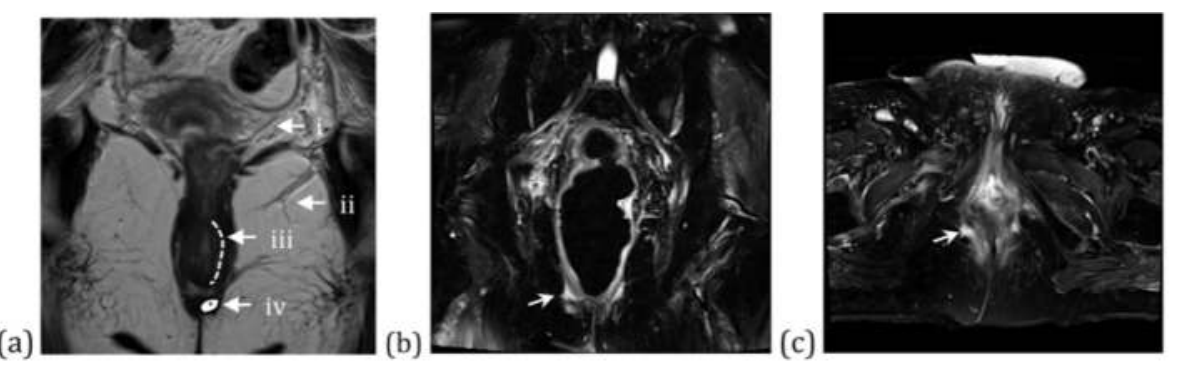

Fistula in Ano The fistulous tracts are categorized using the St Mark's classification system. [5] The majority of fistulae develop secondary to anorectal abscesses.
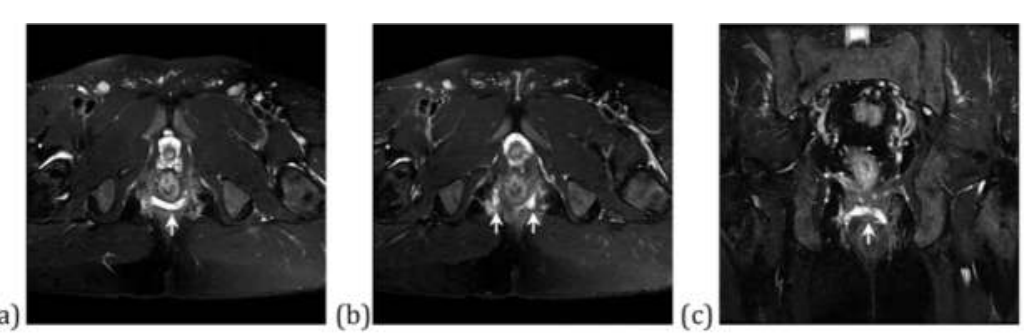

Pilonidal Disease Pilonidal disease refers to a chronic

subcutaneous infection and foreign-body reaction to hairs embedded in the skin in the natal cleft. Sinus tracts can form.
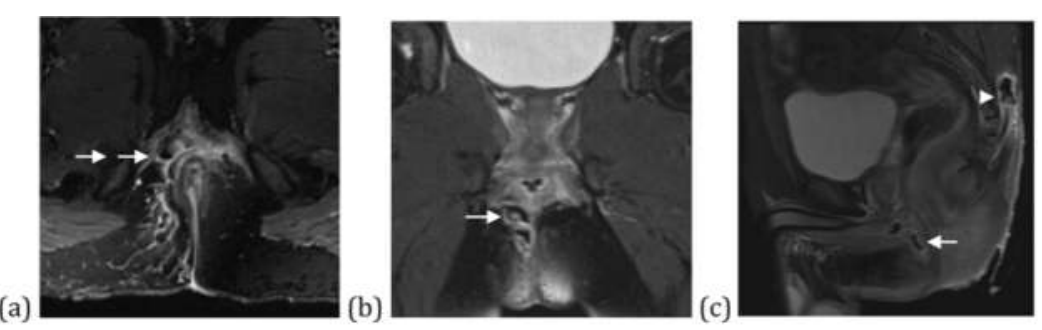

Hidradenitis Suppurativa

Hidradenitis suppurativa is a chronic, recurrent inflammatory process involving the apocrine glands.

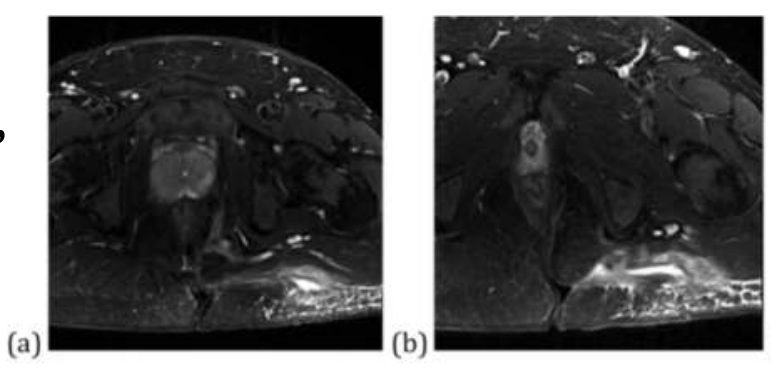

Anal cancer and nearby malignancies

Anal cancer

Squamous cell carcinoma (SCC) is the most common [6]
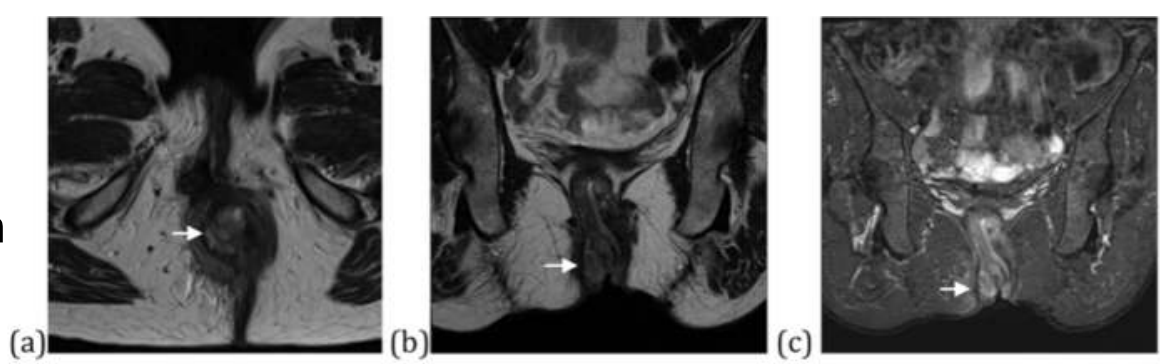

$M R I$ is used in the post treatment follow-up of patients to assess for disease recurrence, after local resection, abdominoperineal resection (Fig. 13) or radiation treatment.

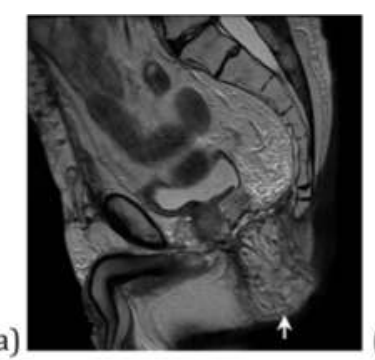

Low rectal cancers Tumours of the lower rectum can invade along the mucosal surface into the anal canal or prolapse through if pedunculated.
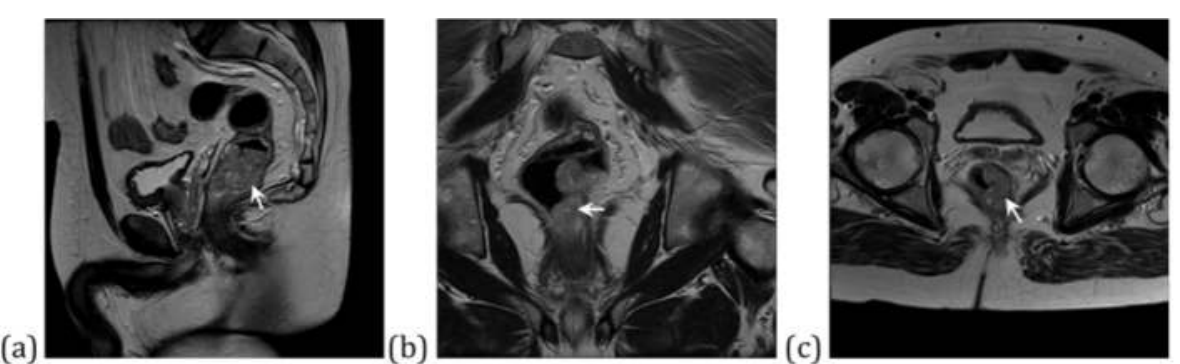

Giant condyloma/Buschke-Lowenstein Verrucous carcinoma of the skin and mucosa is an uncommon type of well-differentiated squamous cell carcinoma [7].
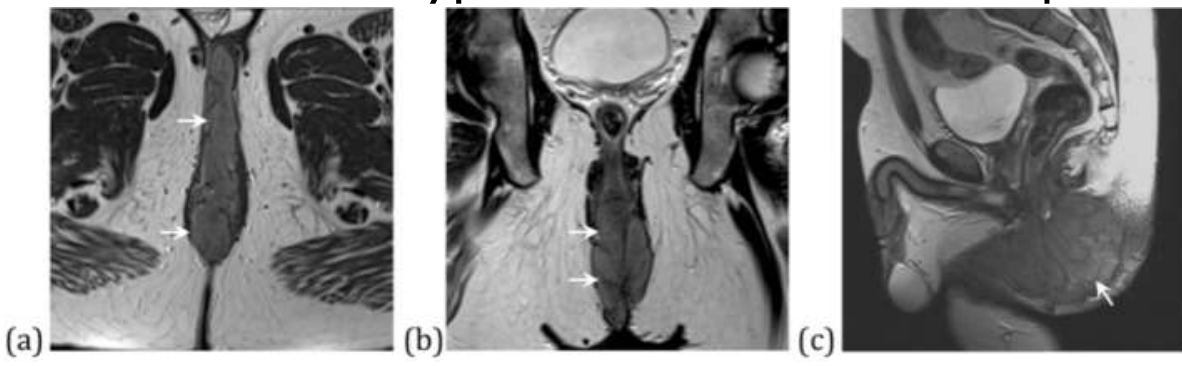

Nearby malignancy within the pelvis

Given that the pelvic

organs lie in close proximity to each other within the relatively small space of the pelvis, direct invasion into nearby structures/organs can be seen in locally advanced

(T4) disease.

\section{Adult Presacral Teratoma}
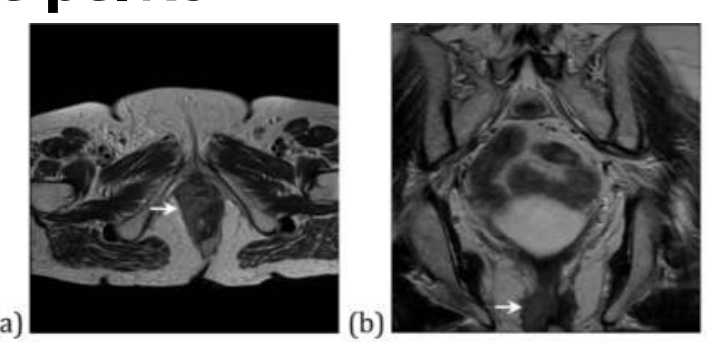

Typically identified in infancy and are extremely rare in adulthood.
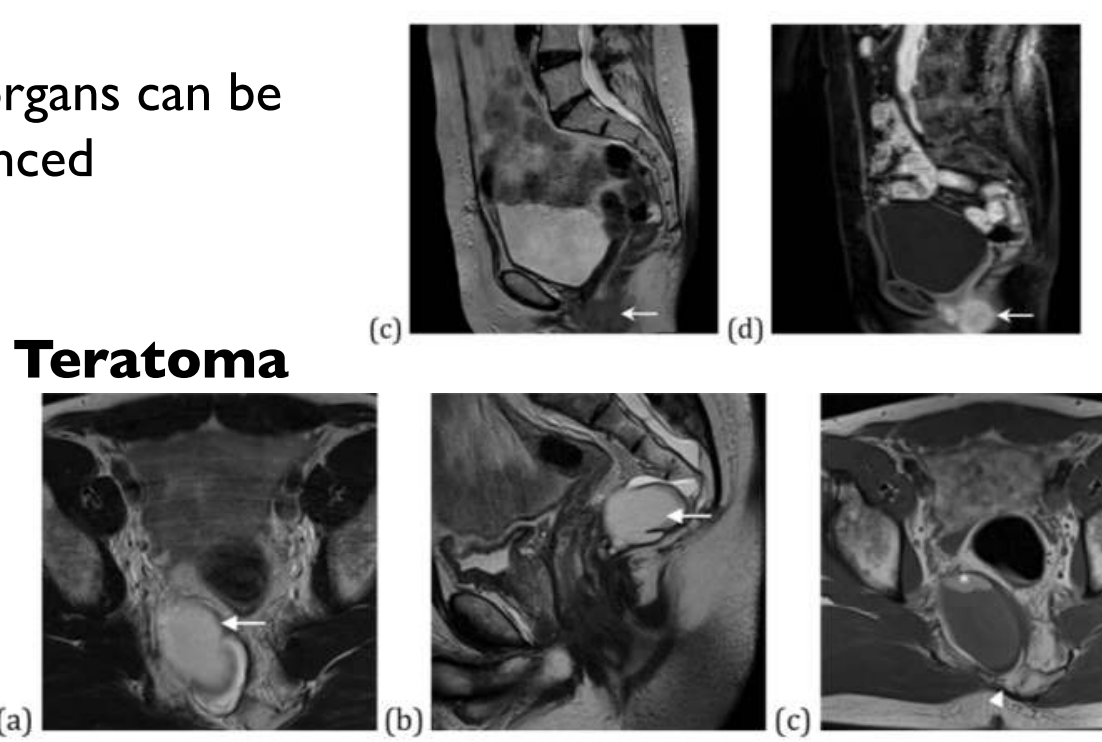

\section{Discussion}

MRI pelvis provides detailed visualisation of the pelvic organs particularly the anal canal. This pictorial review aims to demonstrate the differential diagnosis of diseases of the anal canal; predominantly benign with the inclusion of important malignant diagnoses. While MRI is vital in the accurate staging of anorectal malignancy, it is also extremely helpful in the diagnosis and management of non-malignant conditions including benign developmental, inflammatory and infectious diseases. Furthermore, MRI is sensitive in the detection of post-radiotherapy complications as well as identifying recurrence of malignancy following initial treatment, whether surgical or with chemo/ radiotherapy. 